\title{
The Erdős-Ko-Rado Theorem for Integer Sequences
}

\author{
Peter Frankl \\ CNRS, ER 175 Combinatoire, \\ 54 Bd Raspail, 75006 Paris, France \\ Norihide Tokushige \\ College of Education, Ryukyu University, \\ Nishihara, Okinawa, 903-0213, Japan \\ hide@edu.u-ryukyu.ac.jp
}

\begin{abstract}
For positive integers $n, q, t$ we determine the maximum number of integer sequences $\left(a_{1}, \ldots, a_{n}\right)$ which satisfy $1 \leq a_{i} \leq q$ for $1 \leq i \leq n$, and any two sequences agree in at least $t$ positions. The result gives an affirmative answer to a conjecture of Frankl and Füredi.
\end{abstract}

\section{Introduction}

Let $n, q, t$ be positive integers with $q \geq 2, n \geq t$, and let $[q]:=\{1,2, \ldots, q\}$. Then $\mathcal{H} \subset[q]^{n}$ is a set of integer sequences $\left(a_{1}, \ldots, a_{n}\right), 1 \leq a_{i} \leq q$. We say that $\mathcal{H}$ is $t$-intersecting if any two sequences intersects in at least $t$ positions, more precisely, $\left|\left\{i: a_{i}=a_{i}^{\prime}\right\}\right| \geq t$ holds for all $\left(a_{1}, \ldots, a_{n}\right),\left(a_{1}^{\prime}, \ldots, a_{n}^{\prime}\right) \in \mathcal{H}$. In this paper, we determine the exact value of the following function.

$$
f(n, q, t):=\max \left\{|\mathcal{H}|: \mathcal{H} \subset[q]^{n}, \mathcal{H} \text { is } t \text {-intersecting }\right\} \text {. }
$$

A family $\mathcal{A} \subset 2^{[n]}$ is called $t$-intersecting if $\left|A \cap A^{\prime}\right| \geq t$ holds for all $A, A^{\prime} \in \mathcal{A}$. Define a weighted size of $\mathcal{A}$ by $w(\mathcal{A}):=\sum_{A \in \mathcal{A}}(q-1)^{n-|A|}$. Using a shifting technique, it is not difficult to check the following:

Lemma 1 (Proposition 2 in [5]) $f(n, q, t)=\max _{\mathcal{A}} w(\mathcal{A})$, where $\mathcal{A} \subset 2^{[n]}$ runs over all $t$-intersecting families.

If $q=2$ then $w(\mathcal{A})=|A|$. Thus, $f(n, 2, t)$ is simply the maximal size of $t$-intersecting family $\mathcal{A} \subset 2^{[n]}$, which is given by the Katona Theorem. This case was solved by Kleitman [7].

Let us define a $t$-intersecting family $\mathcal{A}_{r} \subset 2^{[n]}$ by

$$
\mathcal{A}_{r}:=\{A \subset[n]:|A \cap[t+2 r]| \geq t+r\} .
$$


In [5], Frankl and Füredi conjectured $f(n, q, t)=\max _{r \geq 0} w\left(\mathcal{A}_{r}\right)$. If $q \geq t+1$ then the conjecture claims $f(n, q, t)=q^{n-t}$. They showed that this is true if $t \geq 15$.

Now we introduce the full Erdős-Ko-Rado theorem, which was conjectured by Frankl in [4], and proved by Ahlswede and Khachatrian in [1]. Set

$$
\operatorname{AK}(n, k, t, r):=\left|\left\{B \in\left(\begin{array}{c}
{[n]} \\
k
\end{array}\right):|B \cap[t+2 r]| \geq t+r\right\}\right| .
$$

Theorem 1 ([1] $)$ Let $1 \leq t \leq k \leq n$ and $\mathcal{B} \subset\left(\begin{array}{c}{[n]} \\ k\end{array}\right)$ be t-intersecting. If

$$
(k-t+1)\left(2+\frac{t-1}{r+1}\right) \leq n \leq(k-t+1)\left(2+\frac{t-1}{r}\right)
$$

for some $r \in \mathbf{N}$, then $|\mathcal{B}| \leq \mathrm{AK}(n, k, t, r)$.

Using the above result, we prove the following in section 2 .

Theorem 2 Let $q \geq 3$ and set $r:=\left\lfloor\frac{t-1}{q-2}\right\rfloor$. Then $f(n, q, t)=w\left(\mathcal{A}_{r}\right)$ for $n \geq t+2 r$.

Note that

$$
\begin{aligned}
w\left(\mathcal{A}_{r}\right) & =\sum_{j=0}^{n-t-2 r} \sum_{i=t+r}^{t+2 r}\left(\begin{array}{c}
t+2 r \\
i
\end{array}\right)\left(\begin{array}{c}
n-t-2 r \\
j
\end{array}\right)(q-1)^{n-i-j} \\
& =\sum_{j=0}^{n-t-2 r}\left(\begin{array}{c}
n-t-2 r \\
j
\end{array}\right)(q-1)^{n-t-2 r-j} \sum_{i=t+r}^{t+2 r}\left(\begin{array}{c}
t+2 r \\
i
\end{array}\right)(q-1)^{t+2 r-i} \\
& =q^{n-t-2 r} \sum_{i=0}^{r}\left(\begin{array}{c}
t+2 r \\
i
\end{array}\right)(q-1)^{i} .
\end{aligned}
$$

In section 3 , we prove the case $q \geq t+1$ (and $t \geq 1$ ) directly.

Independently, Ahlswede and Khachatrian [2] obtained Theorem 2 as a diametric theorem in Hamming spaces. They used a different method. See [6] or [2] for the history of the problem.

\section{Proof of the theorem}

Throughout this section, we fix $q$ and $t$ and set

$$
r:=\left\lfloor\frac{t-1}{q-2}\right\rfloor=\frac{t-1}{q-2}-\delta .
$$

Let us recall the following easy probabilistic result.

Lemma 2 (Proposition 3 in [5]) For every $\epsilon>0$ the number of sequences $\left(a_{1}, \ldots, a_{n}\right) \in$ $[q]^{n}$ which contain more than $(1+\epsilon)(n / q) 1$ 's or less than $(1-\epsilon)(n / q) 1$ 's is less than $\epsilon q^{n}$ for $n>n_{0}(\epsilon)$.

Choose any sufficiently small positive $\epsilon$, i.e., $0<\epsilon<\epsilon_{0}(q, t)$, and set an open interval $I:=((1-\epsilon)(n / q),(1+\epsilon)(n / q))$. In view of Lemma $1, f(n, q, t) q^{-n}=w(\mathcal{A}) q^{-n}$ for some $t$-intersecting family $\mathcal{A}$. Moreover Lemma 2 gives that

$$
f(n, q, t) q^{-n}<w(\mathcal{B}) q^{-n}+\epsilon
$$

where $\mathcal{B}:=\{B \in \mathcal{A}:|B| \in I\}$. Set $\mathcal{B}(k):=\{B \in \mathcal{B}:|B|=k\}$. 
Case I $0<\delta<1$.

Note that $\delta$ depends only on $t$ and $q$.

Lemma 3 For $k \in I$ and sufficiently large $n$,

$$
(k-t+1)\left(2+\frac{t-1}{r+1}\right) \leq n \leq(k-t+1)\left(2+\frac{t-1}{r}\right)
$$

Proof (2) is equivalent to

$$
(2+(t-1) / r)^{-1} n+t-1 \leq k \leq(2+(t-1) /(r+1))^{-1} n+t-1
$$

Let us show the right half. Since $k<(1+\epsilon)(n / q)$, it is sufficient to show

$$
(1+\epsilon)(n / q) \leq(2+(t-1) /(r+1))^{-1} n+t-1
$$

or

$$
(1+\epsilon)(2+(t-1) /(r+1))<q .
$$

This follows from $q=2+(t-1) /(r+\delta)>2+(t-1) /(r+1)$ and $\epsilon<\epsilon_{0}(q, t)$. One can prove the left half of (3) similarly.

Thus, by the Ahlswede-Khachatrian theorem we have $|\mathcal{B}(k)| \leq \mathrm{AK}(n, k, t, r)$. Therefore,

$$
\begin{aligned}
f(n, q, t) q^{-n} & <q^{-n} \sum_{k \in I} w(\mathcal{B}(k))+\epsilon \\
& \leq q^{-n} \sum_{k \in I} \operatorname{AK}(n, k, t, r)(q-1)^{n-k}+\epsilon \\
& =q^{-n} \sum_{k \in I} \sum_{j=t+r}^{t+2 r}\left(\begin{array}{c}
t+2 r \\
j
\end{array}\right)\left(\begin{array}{c}
n-t-2 r \\
k-j
\end{array}\right)(q-1)^{n-k}+\epsilon \\
& <q^{-n} \sum_{j=t+r}^{t+2 r}\left(\begin{array}{c}
t+2 r \\
j
\end{array}\right) \sum_{k=j}^{n-t-2 r+j}\left(\begin{array}{c}
n-t-2 r \\
k-j
\end{array}\right)(q-1)^{n-k}+\epsilon \\
& =q^{-n} \sum_{j=t+r}^{t+2 r}\left(\begin{array}{c}
t+2 r \\
j
\end{array}\right) \sum_{i=0}^{n-t-2 r}\left(\begin{array}{c}
n-t-2 r \\
i
\end{array}\right)(q-1)^{(n-t-2 r)-i}(q-1)^{t+2 r-j}+\epsilon \\
& =q^{-n} \sum_{j=t+r}^{t+2 r}\left(\begin{array}{c}
t+2 r \\
j
\end{array}\right) q^{n-t-2 r}(q-1)^{t+2 r-j}+\epsilon \\
& =q^{-t-2 r} \sum_{i=0}^{r}\left(\begin{array}{c}
t+2 r \\
i
\end{array}\right)(q-1)^{i}+\epsilon .
\end{aligned}
$$

Hence we have

$$
g(q, t):=\lim _{n \rightarrow \infty} f(n, q, t) q^{-n} \leq q^{-t-2 r} \sum_{i=0}^{r}\left(\begin{array}{c}
t+2 r \\
i
\end{array}\right)(q-1)^{i} .
$$

On the other hand, (1) implies

$$
g(q, t) \geq q^{-t-2 r} \sum_{i=0}^{r}\left(\begin{array}{c}
t+2 r \\
i
\end{array}\right)(q-1)^{i} .
$$


By (4) and (5), we finally have

$$
g(q, t)=q^{-t-2 r} \sum_{i=0}^{r}\left(\begin{array}{c}
t+2 r \\
i
\end{array}\right)(q-1)^{i} .
$$

Now suppose that for some $t$-intersecting family $\mathcal{A} \subset 2^{[n]}$ we have $w(\mathcal{A}) \geq q^{n} g(q, t)+1$. Since $f(n+1, q, t) \geq q f(n, q, t)$ we have

$$
f\left(n^{\prime}, q, t\right) \geq q^{n^{\prime}-n} f(n, q, t) \geq q^{n^{\prime}-n} w(\mathcal{A}) \geq q^{n^{\prime}}\left(g(q, t)+q^{-n}\right),
$$

which implies $\lim _{n^{\prime} \rightarrow \infty} f\left(n^{\prime}, q, t\right) q^{-n^{\prime}} \geq g(q, t)+q^{-n}>g(q, t)$, a contradiction. Thus we must have $w(\mathcal{A}) \leq q^{n} g(q, t)$, and actually $w\left(\mathcal{A}_{r}\right)=q^{n} g(q, t)$. (We need $n \geq t+2 r$ here.) This completes the proof of Case I.

Case II $\delta=0$.

In this case, we have $q=2+\frac{t-1}{r}$.

Lemma 4 For $k \in I$ and sufficiently large $n$,

$$
(k-t+1)\left(2+\frac{t-1}{r+1}\right) \leq n \leq(k-t+1)\left(2+\frac{t-1}{r-1}\right) .
$$

In fact, one can prove

$$
\left(2+\frac{t-1}{r-1}\right)^{-1} n+t-1 \leq(1-\epsilon) \frac{n}{q}<\frac{n}{q}+t-1<(1+\epsilon) \frac{n}{q} \leq\left(2+\frac{t-1}{r+1}\right)^{-1} n+t-1 .
$$

The proof is similar to the proof of Lemma 3 and we omit it. By this lemma, we have

$$
|\mathcal{B}(k)| \leq \max \{\operatorname{AK}(n, k, t, r), \operatorname{AK}(n, k, t, r-1)\} .
$$

If $n=q(k-t+1)$ then $\operatorname{AK}(n, k, t, r)=\operatorname{AK}(n, k, t, r-1)$. Since

$$
\begin{aligned}
\operatorname{AK}(n, k, t, r) & =\sum_{j=0}^{r}\left(\begin{array}{c}
t+2 r \\
t+r+j
\end{array}\right)\left(\begin{array}{c}
n-t-2 r \\
k-t-r-j
\end{array}\right) \\
& =\left(\begin{array}{c}
n-t-2 r \\
k-t-r
\end{array}\right) \sum_{j=0}^{r}\left(\begin{array}{c}
t+2 r \\
t+r+j
\end{array}\right) \prod_{i=1}^{j} \frac{k-t-r-i+1}{n-k-r+i},
\end{aligned}
$$

we have

$$
1=\frac{\operatorname{AK}(n, k, t, r-1)}{\operatorname{AK}(n, k, t, r)}=\frac{(n-t-2 r+2)(n-t-2 r+1)}{(k-t-r+1)(n-k-r+1)} \frac{\sum_{j=0}^{r-1}\left(\begin{array}{c}
t+2 r-2 \\
t+r+j-1
\end{array}\right) \prod_{i=1}^{j} \frac{k-t-r-i+2}{n-k-r+i+1}}{\sum_{j=0}^{r}\left(\begin{array}{c}
t+2 r \\
t+r+j
\end{array}\right) \prod_{i=1}^{j} \frac{k-t-r-i+1}{n-k-r+i}} .
$$

The above ratio tends to

$$
\frac{q^{2}}{(q-1)} \frac{\sum_{j=0}^{r-1}\left(\begin{array}{c}
t+2 r-2 \\
t+r+j-1
\end{array}\right)(q-1)^{-j}}{\sum_{j=0}^{r}\left(\begin{array}{c}
t+2 r \\
t+r+j
\end{array}\right)(q-1)^{-j}}=\frac{q^{2}}{(q-1)} \frac{\sum_{i=1}^{r}\left(\begin{array}{c}
t+2 r-2 \\
i-1
\end{array}\right)(q-1)^{i}}{\sum_{i=0}^{r}\left(\begin{array}{c}
t+2 r \\
i
\end{array}\right)(q-1)^{i}}
$$


as $n \rightarrow \infty$ for fixed $q, t$ and $n=q(k-t+1)$. This proves

$$
q^{2} \sum_{i=1}^{r}\left(\begin{array}{c}
t+2 r-2 \\
i-1
\end{array}\right)(q-1)^{i}=(q-1) \sum_{i=0}^{r}\left(\begin{array}{c}
t+2 r \\
i
\end{array}\right)(q-1)^{i}
$$

Now choose $k \in I$. (Here we do not assume $n=q(k-t+1)$.) Then,

$$
\begin{aligned}
\frac{\mathrm{AK}(n, k, t, r-1)}{\mathrm{AK}(n, k, t, r)} & =\frac{(n-t-2 r+2)(n-t-2 r+1)}{(k-t-r+1)(n-k-r+1)} \frac{\sum_{j=0}^{r-1}\left(\begin{array}{c}
t+2 r-2 \\
t+r+j-1
\end{array}\right) \prod_{i=1}^{j} \frac{k-t-r-i+2}{n-k-r+i+1}}{\sum_{j=0}^{r}\left(\begin{array}{c}
t+2 r \\
t+r+j
\end{array}\right) \prod_{i=1}^{j} \frac{k-t-r-i+1}{n-k-r+i}} \\
& <\frac{n^{2}}{(1-\epsilon)(n / q)(1-(1+\epsilon) / q) n} \frac{\sum_{j=0}^{r-1}\left(\begin{array}{c}
t+2 r-2 \\
t+r+j-1
\end{array}\right) \prod_{i=1}^{j} \frac{(1+\epsilon)(n / q)}{(1-(1+\epsilon) / q) n}}{\sum_{j=0}^{r}\left(\begin{array}{c}
t+2 r \\
t+r+j
\end{array}\right) \prod_{i=1}^{j} \frac{(1-\epsilon)(n / q)}{(1-(1-\epsilon) / q) n}} \\
& =\frac{q^{2}}{(1-\epsilon)(q-1-\epsilon)} \frac{\sum_{i=1}^{r}\left(\begin{array}{c}
t+2 r-2 \\
i-1
\end{array}\right)\left(\frac{q-1-\epsilon}{1+\epsilon}\right)^{i}}{\sum_{i=0}^{r}\left(\begin{array}{c}
t+2 r \\
i
\end{array}\right)\left(\frac{q-1+\epsilon}{1-\epsilon}\right)^{i}} .
\end{aligned}
$$

By (6), the above ratio tends to 1 as $\epsilon \rightarrow 0$. Thus for any $\epsilon^{\prime}>0$ we can conclude that

$$
\operatorname{AK}(n, k, t, r-1)<\left(1+\epsilon^{\prime}\right) \operatorname{AK}(n, k, t, r)
$$

if we choose $\epsilon$ sufficiently small and $n$ sufficiently large, and $k \in I$. Finally we have

$$
\begin{aligned}
f(n, q, t) q^{-n} & <q^{-n} \sum_{k \in I} \max \{\operatorname{AK}(n, k, t, r), \operatorname{AK}(n, k, t, r-1)\}(q-1)^{n-k}+\epsilon \\
& <\left(1+\epsilon^{\prime}\right) q^{-n} \sum_{k \in I} \operatorname{AK}(n, k, t, r)(q-1)^{n-k}+\epsilon \\
& <\left(1+\epsilon^{\prime}\right) q^{-t-2 r} \sum_{i=0}^{r}\left(\begin{array}{c}
t+2 r \\
i
\end{array}\right)(q-1)^{i}+\epsilon .
\end{aligned}
$$

Using the same argument in Case I, we have

$$
g(q, t):=\lim _{n \rightarrow \infty} f(n, q, t) q^{-n}=q^{-t-2 r} \sum_{i=0}^{r}\left(\begin{array}{c}
t+2 r \\
i
\end{array}\right)(q-1)^{i}
$$

and $f(n, q, t)=q^{n} g(q, t)$, which completes the proof of the theorem.

\section{Another approach}

In this section we give a direct proof for the case $q \geq t+1$ using tools developed in [1].

Let $\mathcal{A} \subset 2^{[n]}$. A family $\mathcal{G} \subset 2^{[n]}$ is called a kernel of $\mathcal{A}$ if $\mathcal{A}=\bigcup_{G \in \mathcal{G}} \mathcal{U}(G)$ where $\mathcal{U}(G):=\{F \subset[n]: G \subset F\}$. A rank of $\mathcal{A}$ is defined by

$$
\operatorname{rank}(\mathcal{A}):=\min \left\{\left|\bigcup_{G \in \mathcal{G}} G\right|: \mathcal{G} \text { is a kernel of } \mathcal{A}\right\} .
$$

Theorem 3 Let $\mathcal{A} \subset 2^{[n]}$ be a shifted $t$-intersecting family with $w(\mathcal{A})=f(n, q, t)$. Then $\operatorname{rank}(\mathcal{A}) \leq t+2 r$, where $r:=\left\lfloor\frac{t-1}{q-2}\right\rfloor$.

Since the proof is almost the same as the proof of Lemma 6 in [1], we omit the details. 
Proof (Outline) Choose a shifted, inclusion minimal (i.e., antichain) kernel $\mathcal{G} \subset 2^{[n]}$ of $\mathcal{A}$ satisfying $\operatorname{rank}(\mathcal{A})=\left|\bigcup_{G \in \mathcal{G}} G\right|$. Assume that $\delta>0$ and $M:=t+2 r+\delta=$ $\operatorname{rank}(\mathcal{A})$. Let $\mathcal{G}=\mathcal{G}_{0} \cup \mathcal{G}_{1}, \mathcal{G}_{0}:=\{G \in \mathcal{G}: M \in G\}, \mathcal{G}_{1}:=\mathcal{G}-\mathcal{G}_{0}$, and let

$$
\mathcal{G}_{0}=\mathcal{R}_{t+1} \cup \cdots \cup \mathcal{R}_{M-1},
$$

where $\mathcal{R}_{i}:=\mathcal{G}_{0} \cap\left(\begin{array}{c}{[M]} \\ i\end{array}\right)$. Set

$$
\mathcal{R}_{i}^{\prime}:=\left\{E-\{M\}: E \in \mathcal{R}_{i}\right\} \subset\left(\begin{array}{c}
{[M-1]} \\
i-1
\end{array}\right) .
$$

Then, $E \in \mathcal{R}_{i}^{\prime}, E^{\prime} \in \mathcal{R}_{j}^{\prime}$ and $i+j \neq M+t$ imply $\left|E \cap E^{\prime}\right| \geq t$. Thus we may assume that $\mathcal{R}_{i} \neq \emptyset, \mathcal{R}_{j} \neq \emptyset, i+j=M+t$ for some $i, j$.

Case $\mathbf{I} \quad i \neq j$.

Define

$$
\begin{aligned}
\mathcal{F}_{1} & :=\mathcal{G}_{1} \cup\left(\mathcal{G}_{0}-\left(\mathcal{R}_{i} \cup \mathcal{R}_{j}\right)\right) \cup \mathcal{R}_{i}^{\prime}, \\
\mathcal{F}_{2} & :=\mathcal{G}_{1} \cup\left(\mathcal{G}_{0}-\left(\mathcal{R}_{i} \cup \mathcal{R}_{j}\right)\right) \cup \mathcal{R}_{j}^{\prime}, \\
\mathcal{B}_{i} & :=\mathcal{U}\left(\mathcal{F}_{i}\right) .
\end{aligned}
$$

Then we have

$$
\begin{aligned}
& \mathcal{A}-\mathcal{B}_{1}=\left\{R \cup S: R \in \mathcal{R}_{j}, S \in 2^{[M+1, n]}\right\}, \\
& \mathcal{B}_{1}-\mathcal{A}=\left\{R \cup S: R \in \mathcal{R}_{i}^{\prime}, S \in 2^{[M+1, n]}\right\},
\end{aligned}
$$

and hence

$$
\begin{aligned}
w\left(\mathcal{A}-\mathcal{B}_{1}\right) & =\left|\mathcal{R}_{j}\right|(q-1)^{M-j} q^{n-M}, \\
w\left(\mathcal{B}_{1}-\mathcal{A}\right) & =\left|\mathcal{R}_{i}\right|(q-1)^{M-i+1} q^{n-M} .
\end{aligned}
$$

If $w(\mathcal{A}) \geq w\left(\mathcal{B}_{1}\right)$ and $w(\mathcal{A}) \geq w\left(\mathcal{B}_{2}\right)$ then

$$
\begin{aligned}
\left|\mathcal{R}_{j}\right|(q-1)^{M-j} & \geq\left|\mathcal{R}_{i}\right|(q-1)^{M-i+1} \\
\left|\mathcal{R}_{i}\right|(q-1)^{M-i} & \geq\left|\mathcal{R}_{j}\right|(q-1)^{M-j+1}
\end{aligned}
$$

Thus $1 \geq(q-1)^{2}$, a contradiction.

Case II $\quad i=j=\frac{M+t}{2}=t+r+\frac{\delta}{2}$.

In this case $\delta$ is even and $\delta \geq 2$. Using the same argument in Case I, we may assume that $\mathcal{R}_{\alpha}=\emptyset$ for all $\alpha \neq i$, and $\mathcal{G}=\mathcal{R}_{i} \cup \mathcal{G}_{1}$. The average degree $\bar{d}$ of $\mathcal{R}_{i}^{\prime} \subset\left(\begin{array}{c}{[M-1]} \\ i-1\end{array}\right)$ is given by $\bar{d}=(i-1)\left|\mathcal{R}_{i}\right| /(M-1)$. Therefore we can find $\ell \in[M-1]$ such that $\operatorname{deg}_{\mathcal{R}_{i}^{\prime}}(\ell) \leq \bar{d}$. Define a $t$-intersecting family $\mathcal{T}$ as follows:

$$
\mathcal{T}:=\left\{E \in \mathcal{R}_{i}^{\prime}: \ell \notin E\right\} \subset\left(\begin{array}{c}
{[M-1]-\{\ell\}} \\
i-1
\end{array}\right) .
$$


Then $|\mathcal{T}| \geq\left|\mathcal{R}_{i}^{\prime}\right|-\bar{d}=\frac{M-i}{M-1}\left|\mathcal{R}_{i}\right|$. Let $\mathcal{A}=\mathcal{D}_{1} \cup \mathcal{D}_{2}$ where $\mathcal{D}_{1}:=\mathcal{U}\left(\mathcal{G}_{1}\right), \mathcal{D}_{2}:=$ $\mathcal{U}\left(\mathcal{R}_{i}\right)-\mathcal{D}_{1}$, and let $\mathcal{U}\left(\mathcal{T} \cup \mathcal{G}_{1}\right)=\mathcal{D}_{1} \cup \mathcal{D}_{3}$ where $\mathcal{D}_{3}:=\mathcal{U}(\mathcal{T})-\mathcal{D}_{1}$. Then we have

$$
\begin{aligned}
& w\left(\mathcal{D}_{2}\right)=\left|\mathcal{R}_{i}\right|(q-1)^{M-i} q^{n-M}, \\
& w\left(\mathcal{D}_{3}\right)=|\mathcal{T}|(q-1)^{M-i} q^{n-M+1} \geq \frac{M-i}{M-1}\left|\mathcal{R}_{i}\right|(q-1)^{M-i} q^{n-M+1} .
\end{aligned}
$$

If $w\left(\mathcal{D}_{2}\right) \geq w\left(\mathcal{D}_{3}\right)$ then $1 \geq \frac{M-i}{M-1} \cdot q$. Since $M=t+2 r+\delta$ and $i=t+r+\frac{\delta}{2}$, we have

$$
t+2 r+\delta-1 \geq \frac{2 r+\delta}{2} q
$$

or equivalently,

$$
r \leq \frac{t-1-(q / 2-1) \delta}{q-2}=\frac{t-1}{q-2}-\frac{\delta}{2}
$$

Since $\frac{\delta}{2} \geq 1$ we have $r \leq \frac{t-1}{q-2}-1$, which contradicts a definition of $r$.

Corollary 1 If $q \geq t+1$ then $f(n, q, t)=q^{n-t}$.

Proof Suppose that $\mathcal{A} \subset 2^{[n]}$ is $t$-intersecting and $w(\mathcal{A})=f(n, q, t)$. By Theorem 3 , we may assume $\operatorname{rank}(\mathcal{A}) \leq t+2 r, r:=\left\lfloor\frac{t-1}{q-2}\right\rfloor$. If $q \geq t+2$ then $r=0$, and $f(n, q, t) \leq w\left(\mathcal{A}_{0}\right)=q^{n-t}$.

If $q=t+1$ then $r=1$ and $f(n, q, t) \leq \max \left\{w\left(\mathcal{A}_{0}\right), w\left(\mathcal{A}_{1}\right)\right\}$. In this case we have $w\left(\mathcal{A}_{0}\right)=w\left(\mathcal{A}_{1}\right)=q^{n-t}$.

\section{References}

[1] R. Ahlswede, L.H. Khachatrian. The complete intersection theorem for systems of finite sets. European J. of Combinatorics, 18:125-136, 1997.

[2] R. Ahlswede, L.H. Khachatrian. The diametric theorem in Hamming spaces Optimal anticodes. Adv. in Appl. Math., 20:429-449, 1998.

[3] P. Erdős, C. Ko and R. Rado. Intersection theorems for systems of finite sets. Quart. J. Math. Oxford (2), 12:313-320, 1961.

[4] P. Frankl. The Erdős-Ko-Rado theorem is true for $n=$ ckt. Collq. Math. Soc. János Bolyai, 18:364-375, 1978.

[5] P. Frankl, Z. Füredi. The Erdős-Ko-Rado theorem for integer sequences. SIAM J. Alg. Disc. Math., 1:376-381, 1980.

[6] P. Frankl, Z. Füredi. Beyond the Erdös-Ko-Rado theorem. J. Combin. Theory (A), 56:182-194, 1991.

[7] D. J. Kleitman. On a combinatorial conjecture of Erdős. J. Combin. Theory, 1:209-214, 1966. 